\title{
Variation of nerve fibre layer thickness measurements with age and ethnicity by scanning laser polarimetry
}

\author{
D Poinoosawmy, L Fontana, J X Wu, F W Fitzke, R A Hitchings
}

\begin{abstract}
Aims-Scanning laser polarimetry is a new technique allowing quantitative analysis of the retinal nerve fibre layer in vivo. This technique was employed to investigate the variation of the retinal nerve fibre layer thickness in a group of normal subjects of different ages and ethnic groups.

Methods-150 normal volunteers of different ages and ethnic groups were recruited for this study. Three consecutive 15 degree polarimetric maps were acquired for each subjects. Nerve fibre layer thickness measurements were obtained at 1.5 disc diameters from the optic nerve. Four 90 degree quadrants were identified. Results-The mean nerve fibre layer thickness varied from a minimum of $55.4 \mu \mathrm{m}$ to a maximum of $105.3 \mu \mathrm{m}$, with a mean thickness value of 78.2 (SD 10.6) $\mu \mathrm{m}$. Superior and inferior quadrants showed a comparatively thicker nerve fibre layer than nasal and temporal quadrants. Retinal nerve fibre layer thickness is inversely correlated with age $(p<0.001)$. White people showed thicker nerve fibre layers than Afro-Caribbeans $(p=0.002)$.

Conclusion-The results indicate a progressive reduction of the nerve fibre layer thickness with increasing age. This may be due to a progressive loss of ganglion axons with age as suggested in postmortem studies. A racial difference in nerve fibre layer thickness is present between whites and Afro-Caribbeans.

(Br F Ophthalmol 1997;81:350-354)
\end{abstract}

Glaucoma Unit, Moorfields Eye

Hospital, London

D Poinoosawmy

L Fontana

$\mathrm{J} X \mathrm{Wu}$

R A Hitchings

Institute of

Ophthalmology,

University College

London, London

L Fontana

F W Fitzke

Correspondence to: Mr Roger A Hitchings, Glaucoma Unit, Moorfield Eye Hospital, City Road, London EC1V 2PD.

Accepted for publication 13 January 1997
Aging is accompanied by a reduction of the retinal sensitivity and a deterioration of the visual function. ${ }^{12}$ Some of these changes may be related to a loss of optic nerve fibres with age. Postmortem studies, investigating the optic nerve axon population and its variation with age, have provided considerable evidence of a reduction of the optic nerve axons number with increasing age..$^{3-10}$

As optic nerve axons and the retinal nerve fibre layer (RNFL) are primarily affected in glaucoma, ${ }^{11}{ }^{12}$ age related variations of these structures may be relevant to the assessment of the psychophysical tests commonly used in the diagnosis and follow up of patients with glaucoma, as well as to the morphometrical measurements of the optic nerve head.

In vivo investigation of the RNFL thickness may provide further evidence of ganglion cell axons loss with age.
Although peripapillary RNFL can be directly visualised and imaged in vivo, using red-free fundus ophthalmoscopy and photography, ${ }^{13-15}$ these techniques allow, so far, subjective, qualitative, and semiquantitative evaluation ${ }^{16}{ }^{17}$ of the peripapillary RNFL, but not quantitative thickness measurements.

With the introduction of scanning laser polarimetry, objective measurements of the RNFL thickness may be indirectly estimated in vivo, on the basis of the amount of polarisation change induced by the RNFL on a polarised laser beam passing through it. ${ }^{18} 19$ This technique may allow us to obtain pointwise measurements, in vivo, of the RNFL thickness over a 15 degree retinal area. ${ }^{20}$

The aim of this study was to evaluate whether variations of the RNFL thickness were detectable in a population of normal subjects of different ages and ethnic groups using a scanning laser polarimeter (Nerve Fibre Analyzer, Laser Diagnostic Technologies Inc, San Diego, CA, USA).

\section{Materials and methods}

Basic principles and technical characteristics of the nerve fibre analyser (NFA) are extensively described elsewhere. ${ }^{18-20}$ The NFA consists of a laser source, a polariser, a scanning unit, a polarisation modulator, a corneal compensator, and a polarisation detector. In principle, a polarised, near infrared laser light $(780 \mathrm{~nm})$, travels across the ocular media to the retina and double passes the RNFL before emerging from the eye. The RNFL, behaving as a birefringent medium, changes the state of polarisation of the light passing through it. The polarisation detection unit quantifies the amount of change in the polarisation (retardation) of the light reflected back from the retina, and correlates the amount of retardation with the RNFL thickness. Approximately 1 degree of retardation corresponds to $7.4 \mu \mathrm{m}$ of RNFL thickness measured histopathologically after removal of the cornea. ${ }^{19}$ Travelling to and from the retina polarised light double passes the cornea. A 'corneal compensator' was introduced to correct for the effect of corneal birefringence on the RNFL retardation measurements. ${ }^{20}$

A total of 65536 retinal locations are singly tested, allowing us to create a retardation map corresponding to RNFL thickness over a 15 degree $(256 \times 256$ pixels $)$ retinal area with a spatial resolution of approximately $17 \times 17$ $\mu \mathrm{m} .^{21}$

For this study normal volunteers of different ages and ethnic groups were recruited from Moorfields Eye Hospital staff and their family, patients' spouses, and friends. 


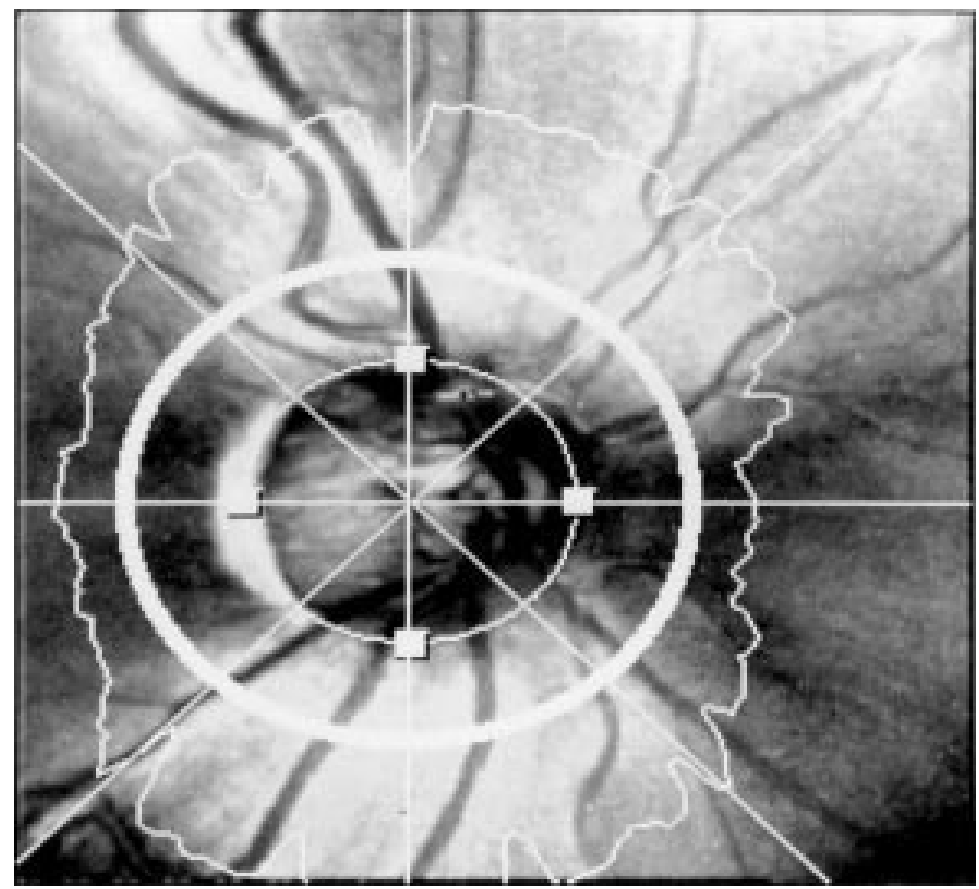

Figure 1 Fifteen degree retardation map of the retinal nerve fibre layer thickness of the right eye of a 35 year old subject. In the grey scale image thicker parts of retinal nerve fibre laver are indicated with lighter shades and thinner parts with darker shades. A circle band of 10 pixels width is centred around the optic disc at 1.5 disc diameter distance. The topographic map is divided in four 90 degree quadrants. Retinal nerve fibre layer thickness is measured within the circle band.

Inclusion criteria were absence of known ophthalmic pathologies affecting the optic nerve or retina, a negative family history for glaucoma, an intraocular pressure $\leq 21 \mathrm{~mm} \mathrm{Hg}$ at the time of examination, and a normal ophthalmoscopic appearance of the optic nerve head at biomicroscopy. Subjects with a refractive error exceeding plus or minus 5 dioptres were excluded from the study.

Informed consent was obtained before imaging with the NFA. For each subject only the right eye was selected for the investigation. In order to improve the accuracy and to test the NFA reproducibility, three images were acquired in sequence and subsequently stored in the computer before the analysis. None of the subjects had pupil dilatation before image acquisition.

Images were acquired by one of the authors (DP), with a 1 year experience of using the NFA. A 15 degree field size was used and the optic nerve head was centred in the middle of the monitor LCD display. Light intensity was adjusted for each subject according to the signal level present on the display.

Image analysis was carried out, using NFA software version 2.11.1, and the following standard settings. RNFL thickness was measured over a circular band of 10 pixels width and 1.5 disc diameters. The band was centred around the optic nerve head. Four 90 degrees quadrants divided the measurements in four sectors: superior 45-135 degrees, inferior 225315 degrees, nasal 135-225, and temporal 45-315 degrees (Fig 1).

RNFL thickness values were obtained from the separate analysis of the three images acquired for each subject. NFA reproducibility
Table 1 Subject distribution according to age

\begin{tabular}{lll}
\hline Age group & Age (years) & $n$ \\
\hline 1 & $\leq 10$ & 10 \\
2 & $11-20$ & 11 \\
3 & $21-30$ & 22 \\
4 & $31-40$ & 24 \\
5 & $41-50$ & 15 \\
6 & $51-60$ & 30 \\
7 & $61-70$ & 19 \\
8 & $\geq 71$ & 19 \\
\hline
\end{tabular}

was evaluated by computing the mean, standard deviation, and coefficient of variation $(\mathrm{COV}=\mathrm{SD} /$ mean $\times 100)$ of the RNFL thickness values obtained for each subject. Analysis of variance (ANOVA) was employed to test the null hypothesis of no difference for the variation of the measurements between subjects of different ages. The NFA measurements and age data were regressed as continuous variables. Ethnic groups were compared using the Mann-Whitney U test. For all the tests a $p$ value less than 0.05 was considered significant.

\section{Results}

A total of 150 volunteers of four ethnic groups were recruited for this study. Of these 59 were white (39.3\%), 51 Afro-Caribbean (34\%), 24 Asian (16\%), and 16 Chinese (10.7\%). Ages ranged between 5 to 90 years with a mean (1 SD) age of 43.9 (20.9) years. Male/female ratio was $1.3(85 / 65)$. Patients were subdivided in eight groups according to age (Table 1).

The NFA software automatically calculated the average RNFL thickness measured within the 10 pixels width circular band. Thickness values of the four 90 degree quadrantssuperior, inferior, nasal, and temporal-were recorded separately. Mean RNFL thickness values for the three images acquired were computed for each subject.

The mean RNFL thickness varied from a minimum of $55.4 \mu \mathrm{m}$ to a maximum of 105.3 $\mu \mathrm{m}$, with a mean thickness of $78.2(10.6) \mu \mathrm{m}$. Similar values of RNFL thickness were found between men and women, 78.4 (11.6) and $77.8(9.2) \mu \mathrm{m}$, respectively $(\mathrm{p}=0.91)$. Mean RNFL thickness values for age groups are reported in Table 2 .

Reproducibility was assessed by computing the COV of the three average RNFL thickness values obtained for each subject. The COV varied from a minimum of $0.1 \%$ to a maximum of $19.7 \%$ with a median value of $3.9 \%$ (Table 2). The COV did not vary significantly among the age groups $(\mathrm{p}=0.59)$.

Table 2 Mean retinal nerve fibre layer (RNFL) thickness ( $\mu \mathrm{m})$ by age groups

\begin{tabular}{llll}
\hline Age group & $\begin{array}{l}\text { RNFL } \\
\text { (mean (1 SD)) }\end{array}$ & $\begin{array}{l}\text { RNFL } \\
\text { (range) }\end{array}$ & $\begin{array}{l}\text { COV (\%) } \\
\text { (mean (1 SD)) }\end{array}$ \\
\hline 1 & $95.3(5.6)$ & $83.5-103.3$ & $3.1(0.8)$ \\
2 & $90(7.7)$ & $75.5-98.6$ & $3.9(1.5)$ \\
3 & $80.2(7.4)$ & $67.3-92.4$ & $4.3(3.8)$ \\
4 & $77.6(9.1)$ & $60.3-96.9$ & $5.7(4.3)$ \\
5 & $80(9.1)$ & $69.2-105.3$ & $4.6(2.0)$ \\
6 & $75.3(8)$ & $59.3-95$ & $4.1(2.5)$ \\
7 & $74.5(10.7)$ & $61.1-97.6$ & $5.3(5.4)$ \\
8 & $67.5(5.6)$ & $55.4-77.5$ & $5.6(3.9)$ \\
\hline
\end{tabular}

Measurements are derived from retardation measurements, 1 degree retardation corresponds to approximately $7.4 \mu \mathrm{m}$. 
When the mean RNFL thickness and the age were plotted for each subject, the RNFL thickness was seen to decrease significantly ( $\mathrm{p}<$ 0.001) with increasing age (Fig 2). This

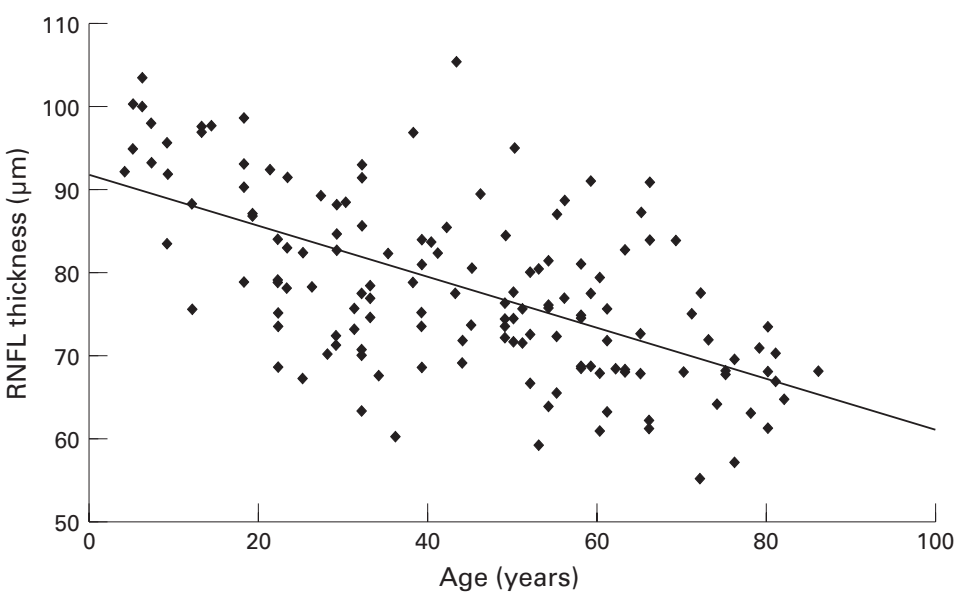

Figure 2 Linear regression analysis of the retinal nerve fibre layer thickness and age. $R=$ $0.5938, R^{2}=0.3526, S E 8.59324, p=<0.001, n=150$. The analysis indicates a retinal nerve fibre layer thickness decay of $0.38 \mu \mathrm{m} /$ year.

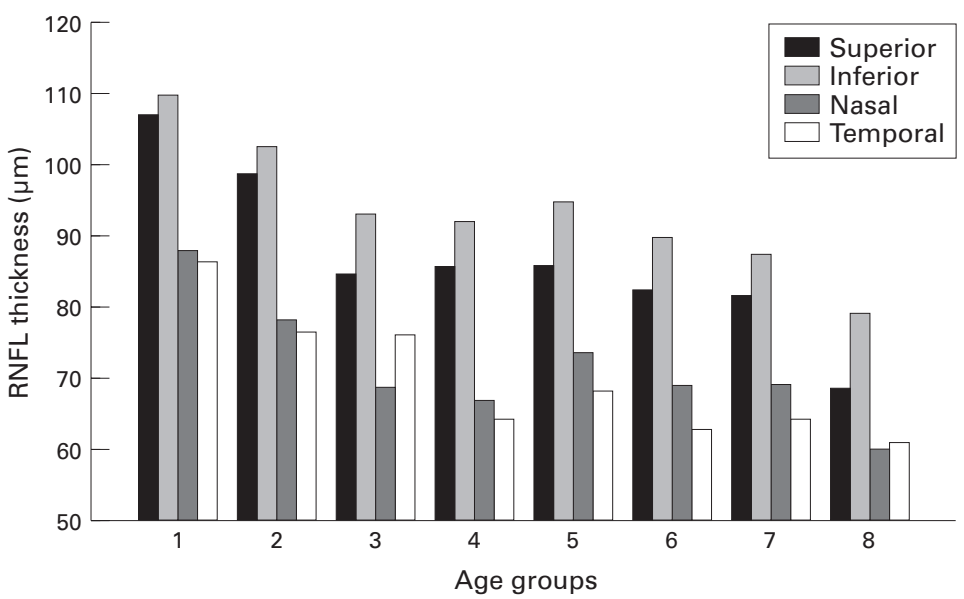

Figure 3 Mean retinal nerve fibre layer thickness by quadrants for the different age groups.

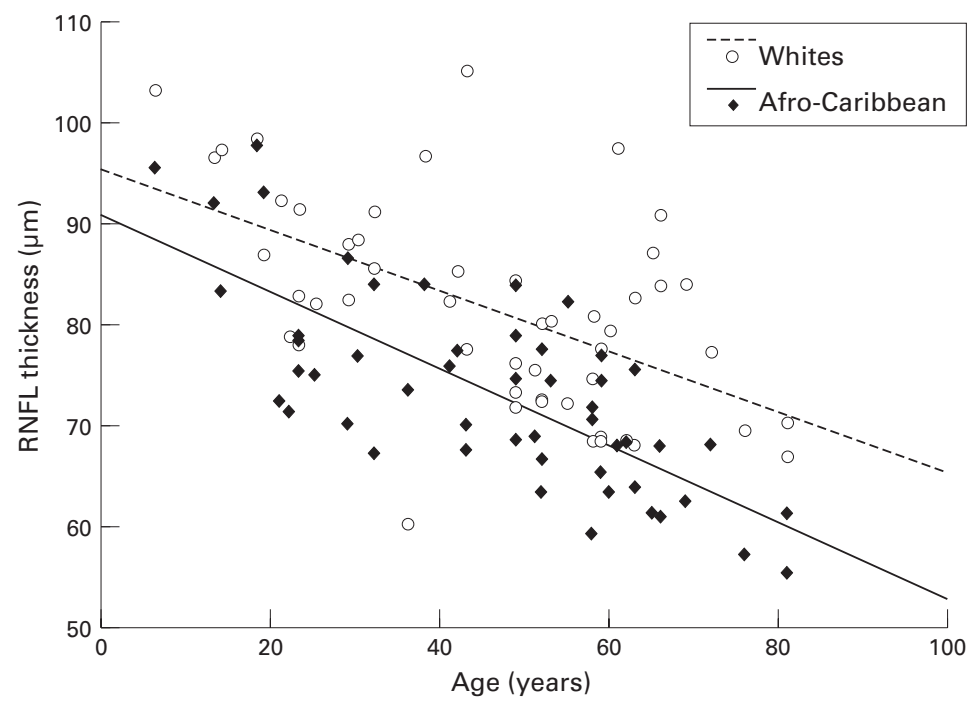

Figure 4 Linear regression analysis of the retinal nerve fibre layer thickness and age for white and Afro-Caribbean groups. $R=0.6499, R^{2}=0.4224, S E 8.20026, p<0.001, n=$ 59 for whites and $R=0.7588, R^{2}=0.5758, S E 6.37953, p<0.001, n=51$ for Afro-Caribbeans. applied to all the quadrants, but more noticeably to the inferior and superior quadrants (Fig 3). The topographical distribution of the RNFL thickness among the four quadrants investigated was similar in all age groups. Superior and the inferior quadrants showed highest RNFL thickness values; temporal, and nasal quadrants revealed comparatively thinner values (Fig 3). In order to assess whether the RNFL thickness presented similar variations with age in subjects of different ethnic groups, linear regression analysis was carried out for the white and the Afro-Caribbean groups. Asian and Chinese groups were excluded as the number of subjects in these groups was considered to be too small for the analysis. Both white and Afro-Caribbean groups showed a significant reduction in mean RNFL thickness with increasing age $(p<0.001$ for both groups) (Fig 4). Although the rate of decay of RNFL thickness with age was similar in the two groups, in this study AfroCaribbeans generally showed lower values of RNFL thickness compared with whites. Mean RNFL thickness and age groups for both whites and Afro-Caribbeans is represented in Table 3. The RNFL thickness in whites ranged from 60.3 to $105.3 \mu \mathrm{m}$ with a mean of 79.7 (10.7) $\mu \mathrm{m}$. The RNFL thickness in AfroCaribbeans ranged from 71.8 to $94.9 \mu \mathrm{m}$ with a mean of $79.6(7.9) \mu \mathrm{m}$. The difference between the two ethnic groups was statistically significant $(p=0.002)$.

\section{Discussion}

Investigations on the effect of age on the optic nerve fibre population in normal individuals has been the subject of various histological studies. ${ }^{3-10}$ Dolman and colleagues ${ }^{3}$ described a diminishing density of axons in the optic nerve sections of elderly subjects, accompanied by an increasing presence of fibrous tissue. Balazi and colleagues, ${ }^{4}$ quantitatively studied the effect of aging on the optic nerve fibre population, reporting a mean loss of 5.637 fibres per year, corresponding to a total loss of about $35 \%$ of the optic nerve fibre population in a lifespan. Subsequent studies, ${ }^{5-10}$ using automated techniques of analysis of the specimens, more accurately determined the number of optic nerve fibres in normal subjects and its variation with age. Besides the differences in mean nerve fibre counts in these studies, a decline of the optic nerve fibre population with increasing age was found with a rate of loss of 4000-5000 fibres per year. ${ }^{46810}$

As the optic nerve axon numbers decrease with age, variations of the RNFL thickness may also be expected.

Evaluation of the RNFL in vivo has been so far limited to qualitative or semiquantitative assessments of red-free fundus photographs ${ }^{16} 17$ or, more recently, to images of the RNFL acquired with the scanning laser ophthalmoscope. ${ }^{22}$ Jonas et $a l^{23}$ correlated the visibility of RNFL, semiquantitatively assessed on red-free photographs, with the age of a large number of normal subjects, describing a reducing visibility of the RNFL with increasing 
Table 3 Mean retinal nerve fibre layer (RNFL) thickness ( $\mu m)$ by age groups in white and Afro-Caribbean groups

\begin{tabular}{|c|c|c|c|c|c|c|c|}
\hline \multirow[b]{2}{*}{ Age group } & \multirow{2}{*}{$\begin{array}{l}\text { White } \\
(n)\end{array}$} & \multirow{2}{*}{$\begin{array}{l}\text { Afro-Caribbean } \\
\text { (n) }\end{array}$} & \multicolumn{2}{|l|}{ RNFL White } & \multicolumn{2}{|c|}{ RNFL Afro-Caribbean } & \multirow[b]{2}{*}{$p$ Value ${ }^{\star}$} \\
\hline & & & (mean (1 SD)) & (range) & $($ mean $(1 S D))$ & (range) & \\
\hline 1 & 1 & 5 & - & - & $92.5(5.5)$ & $83.5-97.9$ & - \\
\hline 2 & 4 & 3 & $95(5.3)$ & 87-98.6 & $80.4(5.8)$ & $75.5-86.5$ & 0.05 \\
\hline 3 & 8 & 9 & $84.7(5.4)$ & $78.2-92.4$ & $75.2(5.9)$ & $67.3-84.1$ & 0.01 \\
\hline 4 & 5 & 12 & $84.5(14.1)$ & $60.3-96.9$ & $74.2(6.2)$ & $63.5-84.0$ & 0.06 \\
\hline 5 & 8 & 1 & $82.1(10.5)$ & $72.2-105.3$ & - & - & - \\
\hline 6 & 12 & 9 & $74.4(4.5)$ & $68.6-81.0$ & $69.8(6.4)$ & $59.3-77.6$ & 0.11 \\
\hline 7 & 9 & 8 & $82.5(9.6)$ & $68.2-97.6$ & $66(4.9)$ & $61.1-75.7$ & $<0.001$ \\
\hline 8 & 12 & 4 & 69 (3.6) & $63.7-77.5$ & $60.6(5.6)$ & $55.4-68.2$ & 0.02 \\
\hline
\end{tabular}

Measurements are derived from retardation measurements, 1 degree retardation corresponds to approximately $7.4 \mu \mathrm{m}$. ${ }^{\star}$ Mann-Whitney U test for the comparison of groups.

age. As the visibility of the RNFL depends on its thickness, the optical variation of this structure with age may be related to loss of the retinal nerve fibres.

The scanning laser polarimeter was developed to measure the RNFL thickness in vivo by adopting its birefringent property of changing the state of polarisation of a laser light passing through it. In principle, the amount of change in polarisation (retardation) is thought to be proportional to the thickness of the RNFL at any tested point. ${ }^{18} \mathrm{~A}$ histopathological study ${ }^{19}$ validating retardation measurements of the RNFL, acquired using the NFA, suggested a good correlation between histological and retardation measurements.

Using the scanning laser polarimeter, we intended to investigate quantitatively in vivo the RNFL thickness in a population of normal volunteers. The results indicate a considerable variation of RNFL thickness among the subjects studied, even of the same age groups. A significant reduction of the RNFL thickness was found with increasing age, with a decay of $0.38 \mu \mathrm{m} /$ year. Furthermore, retardation measured at different quadrants showed variations corresponding to some of the known topographical characteristics of RNFL described in primate eyes. ${ }^{24}{ }^{25}$ Superior and inferior quadrants presented the highest values of RNFL thickness, whereas temporal and nasal quadrants showed consistently lower thickness values.

Similar findings were also described in a previous studies using the scanning laser polarimeter to investigate the RNFL of normal subjects and glaucomatous patients. ${ }^{21}$ Using the same instrument, Chi et $a l^{26}$ calculated that the RNFL thickness decreases with a rate of $0.2 \mu \mathrm{m} /$ year.

The RNFL is not the only birefringent structure of the eyes, scanning laser polarimetric measurements are influenced by the anterior segment birefringence (cornea and lens). Although this source of artefacts should be appropriately compensated in the $\mathrm{NFA},{ }^{20}$ we cannot exclude that possible variation of the anterior optical media birefringence with age may have somehow influenced our findings.

Both white and Afro-Caribbean groups showed a similar reduction of RNFL thickness with age. However, for the same age groups, RNFL in whites was often found to be thicker than in Afro-Caribbeans. This ethnic difference may be explained by a systematic variation in site of measurements between whites and Afro-Caribbeans as a result of a difference in optic disc size between the two ethnic groups. ${ }^{28}$ We used the NFA to measure the RNFL thickness at 1.5 disc diameters. This could result in measurements being made further away from the optic nerve head in eyes with large optic discs, with a resulting difference in retardation measurements, as the RNFL thickness diminishes from the optic disc towards the periphery. ${ }^{26}$ As in this study no optic disc measurements were carried out, we are unable to exclude the possibility that ethnic differences in RNFL thickness may be due to difference in disc size.

However, our findings are supported by those of Varma et $a l^{27}$ who noted in a study of urban white and black Americans that for optic discs of the same size blacks had a smaller neuroretinal rim area than whites.

In conclusion, our study further supports the evidence of a progressive decay of ganglion fibres with age. Furthermore, a difference in RNFL thickness between white and AfroCaribbeans, measured by scanning laser polarimetry, may indicate ethnic differences in nerve fibres in the normal population.

1 Katz J, Sommer A. Asymmetry and variation in the normal hill of vision. Arch Ophthalmol 1986;104:65-8.

2 Heijl A, Lindgren G, Olsson J. Normal variability of static perimetric threshold values across the central visual field.
pelic Arch Ophthalmol 1987;105:1544-9.

3 Dolman CL, McCormick AQ, Drance SM. Ageing and the optic nerve. Arch Ophthalmol 1980;98:2053-8.

4 Balazi AG, Rootman J, Drance SM, Shulzer M, Douglas GR. The effect of age on the nerve fibre population of the optic nerve. Am f Ophthalmol 1984;97:760-6.

5 Johnson BM, Miao M, Sadum AA. Age-related decline of human optic nerve axon populations. Age 1987;10:5-9.

6 Mikelberg FS, Drance SM, Schuler M, Yidegiligne HM, Weis M. The normal human optic nerve. Ophthalmology 1989;96:1325-8.

7 Repka MX, Quigley HA. The effect of age on normal human optic nerve fibre number and diameter. Ophthalmology 1989;96:26-32.

8 Jonas JB, Muller-Berg JA, Schlotzer-Schrehardt UM, Nauman GOH. Histomorphometry of the human optic nerve. Invest Ophthalmol Vis Sci 1990;31:736-44

9 Mikelberg FS, Haile MY, White VA, Schulzer M. Relation Mikelberg FS, Haile MY, White VA, Schulzer M. Relation
between optic nerve axon number and axon diameter to between optic nerve axon number and axon diam
scleral canal area. Ophthalmology 1991;98:60-3.

10 Jonas JB, Schmidt AM, Muller-Bergh JA, SchlotzerSchrehardt UM, Naumann GOH. Human optic nerve fibre count and optic disc size. Invest Ophthalmol Vis Sci 1992;33:2012-8.

11 Quigley HA, Green WR. The histology of human glaucoma cupping and optic nerve damage: Clinicopathologic correlation in 21 eyes. Ophthalmology 1979;86:1803-27

12 Sommer A, Katz J, Quigley HA, Miller NR, Robin AL, Richter RC, et al. Clinically detectable nerve fibre atrophy precedes the onset of glaucomatous field loss. Arch Ophthalmol 1991;109:77-83

13 Airaksinen PJ, Nieminen H. Retinal nerve fibre layer

14 Miller NR, George TW. Monochromatic (red-free) photography and ophthalmoscopy of the peripapillary retinal
ging graphy and ophthalmoscopy of the peripapillary retinal
nerve fibre layer. Invest Ophthalmol Vis Sci 1978;17:1121-4 nerve fibre layer. Invest Ophthalmol Vis Sci 1978;17:1121-4
15 Sommer A, D'Anna SA, Kues HA, George T. Highresolution photography of the retinal nerve fibre layer. $\mathrm{Am}$ f Ophthalmol 1983;96:535-9 
16 Airaksinen PJ, Drance SM, Douglas GR, Mawson DK, Nieminen H. Diffuse and localized nerve fibre loss in glaucoma. Am f Ophthalmol 1984:98:566-71.

17 Quigley HA, Reacher M, Katz J, Strahlman E, Gilbert D, Scott R. Quantitative grading of nerve fibre layer photography. Ophthalmology 1993;100:1800-7.

18 Dreher AW, Reiter K, Wienreg R. Spatially resolved birefringence of retinal nerve fibre layer assessed with retinal laser ellipsometer. Appl Optics 1992;31:3730-5.

19 Weinreb RN, Dreher AW, Coleman A. Histopathologic validation of Fourier-ellipsometry measurements of retinal fibre layer thickness. Arch Ophthalmol 1990;108:557-60.

20 Dreher AW, Reiter K. Retinal laser ellipsometry: a new method for measuring the retinal nerve fibre layer thickness distribution. Clin Vis Sci 1992;7:481-8.

21 Weinreb RN, Shakiba S, Zangwill L. Scanning laser polarimetry to measure the nerve fibre layer of normal and
glaucomatous eyes. Am $\mathcal{F}$ Ophthalmol 1995;119:627-36.
22 Miglior S, Rossetti L, Brigatti L, Bujtar E, Orzalesi N. Reproducibility of retinal nerve fibre layer evaluation by
dynamic scanning laser ophthalmoscope. Am f Ophthalmol 1994;118:16-23.

23 Jonas JB, Nhung XN, Nauman GOH. The retinal nerve fibre layer in normal eyes. Ophthalmology 1989;96:627-32.

24 Radius RL. Thickness of the retinal nerve fibre layer in primate eyes. Arch Ophthalmol 1980;98:1625-9.

25 Quigley HA, Addicks EM. Quantitative studies of retinal nerve fibre layer defects. Arch Ophthalmol 1982;100:807-14.

26 Chi Q-M, Tomita G, Inazumi K, Hayakawa T, Ido T, Kitasawa $\mathrm{H}$. Evaluation of the effect of aging on the retinal nerve fiber layer thickness usin scanning laser polarimetry. f Glaucoma 1995;4:406-13.

27 Varma R, Tielsch JM, Quigley HA, Hilton SC, Katz J, Spaeth GL, Sommer A. Race, age, gender and refractive error related differences in the normal optic disc. Arch Ophthalmol 1994;112:1068-76. 